\title{
microRNA-338-3p functions as a tumor suppressor in human non-small-cell lung carcinoma and targets Ras-related protein 14
}

\author{
JIANGTAO SUN, XIAOSHANG FENG, SHEGAN GAO and ZHONGYUE XIAO \\ Institute of Cancer Research, The First Affiliated Hospital of Henan University of Science Technology, \\ Luoyang, Henan 471003, P.R. China
}

Received November 11, 2013; Accepted May 28, 2014

DOI: $10.3892 / \mathrm{mmr} .2014 .2880$

\begin{abstract}
RNAs (miRNAs) have been demonstrated to be important gene regulators with critical roles in diverse biological processes, including tumorigenesis. Accumulating evidence suggests that miR-338-3p exerts a tumor suppressor role and is downregulated in tumors, including gastric cancer and colorectal carcinoma. However, the role of miR-338-3p in lung cancer, particularly non-small-cell lung carcinoma (NSCLC), has remained elusive. In the present study, the expression levels of miR-338-3p in NSCLC tissues were compared with those of matched normal tissues by use of polymerase chain reaction analysis. miR-338-3p was shown to be downregulated in NSCLC tissues, and the expression levels of miR-338-3p were significantly correlated with NSCLC cancer differentiation, pathological stage and lymph-node metastasis. Ectopic miR-338-3p expression significantly suppressed the in vitro proliferation and colony formation of NSCLC cells and enhanced apoptosis. Of note, ectopic miR-338-3p expression significantly inhibited Ras-related protein 14 (RAB14) mRNA and protein expression, and reduced luciferase reporter activity containing the RAB14 3'-untranslated region through the first binding site. These findings suggested that miR-338-3p regulated the survival of NSCLC cells partially through the downregulation of RAB14. Therefore, targeting the miR-338-3p/RAB14 interaction may serve as a novel therapeutic application to treat NSCLC patients.
\end{abstract}

\section{Introduction}

Lung cancer is the most frequent cause of cancer-associated mortality worldwide. Non-small-cell lung carcinoma (NSCLC), which is usually diagnosed at an advanced stage,

Correspondence to: Dr Xiaoshang Feng, Institute of Cancer Research, The First Affiliated Hospital of Henan University of Science Technology, 24 Jinghua Road, Luoyang, Henan 471003, P.R. China

E-mail: samfeng1377@163.com

Key words: microRNA-338-3p, non-small-cell lung carcinoma, Ras-related protein 14, cell growth, cell apoptosis accounts for $70-80 \%$ of lung cancer cases (1). Despite recent advances in clinical and experimental oncology, the prognosis of NSCLC patients, particularly those with advanced disease, remains poor (2). Therefore, investigation of the molecular mechanisms underlying NSCLC tumorigenesis may aid in the development of novel therapeutic targets and strategies for the treatment of the malignancy.

microRNAs (miRNAs) are small non-coding RNAs, $\sim 22 \mathrm{nt}$ in length, that bind to partially complementary recognition sequences of mRNA, causing either degradation or inhibition of translation, thus effectively silencing the mRNA target (3). As determined by these mechanisms, miRNAs have been reported to be involved in various biological processes, including differentiation, morphogenesis and tumorigenesis (4-6). Studies have revealed that miRNAs exert oncogenic or tumor suppressor roles in the etiology and pathogenesis of cancer by targeting tumor suppressors or oncogenes $(7,8)$. One target gene may be regulated by multiple miRNAs and one miRNA may regulate multiple target genes, which results in the formation of complex regulation networks in tumorigensis (9). Therefore, analysis of the roles of miRNAs in NSCLC cellular transformation and tumorigenesis remains at an early stage. Thus, it is important to understand the characterization of the miRNA pathways and the underlying molecular mechanisms associated with NSCLC progression and development by identification of miRNA targets.

In the present study, miR-338-3p expression levels in NSCLC tissues were compared with those of matched normal adjacent tissues. The functions of miR-338-3p in A549 and SPC-A1 NSCLC cells were further analyzed by MTT and colony formation assays. The effects of miR-338-3p on apoptosis and Ras-related protein 14 (RAB14) expression levels in A549 cells were also examined.

\section{Materials and methods}

Tissue samples. A total of 32 patients diagnosed with primary NSCLC at the First Affiliated Hospital of Henan University of Science Technology (Henan, China) were included in the present study. None of these patients had received chemotherapy and radiotherapy prior to surgery. Tumor and corresponding non-tumor lung tissue samples were collected and rapidly frozen in liquid nitrogen and stored at $-80^{\circ} \mathrm{C}$. The present study was approved by the ethics committee of 
the First Affiliated Hospital of Henan University of Science Technology (Luoyang, China) and full informed consent was provided by all of the patients involvedprior to sample collection.

Cell culture. The A549 and SPC-A1 NSCLC cell lines (American Type Culture Collection, Manassas, VA) were cultured in RPMI 1640 (Gibco-BRL, Carlsbad, CA, USA) medium supplemented with $10 \%$ fetal bovine serum, $100 \mathrm{U} / \mathrm{ml}$ penicillin and $100 \mathrm{mg} / \mathrm{ml}$ streptomycin (Invitrogen Life Technologies, Carslbad, CA, USA) in humidified air at $37^{\circ} \mathrm{C}$ with $5 \% \mathrm{CO}_{2}$.

Bioinformatics. The miRNA targets, which were predicted by computer-aided algorithms were obtained from PicTar (http://pictar.mdc-berlin.de/cgi-bin/new_PicTar_vertebrate.cgi.), TargetScan (http://www.targetscan.org) and miRBase (http://microrna.sanger.ac.uk/cgi-bin/targets/v5/search.pl) databases.

Polymerase chain reaction (PCR). A TaqMan miRNA-assay kit was obtained from Applied Biosystems (Foster City, CA, USA) for the detection of mature miR-338-3p expression levels. According to the manufacturer's instructions, the $2^{-\Delta \Delta \mathrm{Ct}}$ method was used in conjunction with the RNU6B gene as a control for normalization. All experiments were performed in triplicate and repeated once. For analysis of RAB14 mRNA expression levels, total RNA was extracted from the cells and was reverse-transcribed using reverse transcription (RT)-PCR kits (Applied Biosystems) with an oligo $\mathrm{d}(\mathrm{T}) 16$ primer under standard conditions. RAB14-specific primers were designed as follows: Forward, 5'-CGCTCGAGATGGCAACTGCACCATACAAC-3' and reverse, 5'-CGGAATTCCTAGCAGCCACAGCCTTCTC-3'. To verify the integrity of RAB14 expression, the GAPDH gene served as an internal control; the sequences of the GAPDH primers were as follows: Sense, 5'-GGAGTCAACGGATTTGGTCG-3' and antisense, 5'-CATCGCCCCACTTGATTTTG-3'. The following PCR conditions were used: 30 cycles of denaturation at $94^{\circ} \mathrm{C}$ for $30 \mathrm{sec}$, annealing at $56^{\circ} \mathrm{C}\left(58^{\circ} \mathrm{C}\right.$ for $\left.\mathrm{GAPDH}\right)$ for $30 \mathrm{sec}$ and extension at $72^{\circ} \mathrm{C}$ for $30 \mathrm{sec}$. Each PCR product was separated by $1.5 \%$ agarose gel electrophoresis and visualized by ethidium bromide staining (CAS:1239-45-8; Sigma, Munich, Germany).

Western blot analysis. The cell protein lysates were separated by $10 \%$ SDS-PAGE, electrophoretically transferred to polyvinylidene difluoride membranes (Roche Diagnostics $\mathrm{GmbH}$, Mannheim, Germany), then detected with rabbit polyclonal anti-cleaved caspase-3 (1:1,000) and rabbit polyclonal anti-RAB14 $(1: 2,000)$ antibodies for $1 \mathrm{~h}$ at room temperature. Following three rinses (5 min each), the membranes were incubated with secondary antibodies $(1: 5,000)$ for $1 \mathrm{~h}$ at room temperature using mouse anti-GAPDH monoclonal antibody. ECL Plus substrate (Thermo Fisher Scientific, Rockford, IL, USA) was then used for detection. Lab Works Image Acquisition and Analysis software (UVP version 3.0; LLC, Upland, CA, USA) was used to quantify band intensities. The primary and secondary antibodies were purchased from Univ-bio, Inc. (Shanghai, China).
Table I. Characteristics of lung cancer patients.

\begin{tabular}{lr} 
Features & Cases \\
\hline Tumor diameter $(\mathrm{cm})$ & \\
$<5$ & 17 \\
$\geq 5$ & 15
\end{tabular}

Family history of tumor

Negative

Positive

Age

$\geq 60$

$<60$

Differentiation

Good and moderate

Poor or no differentiation

TNM stage

I+II

III+IV

Lymphatic metastasis

$\mathrm{pN}(\mathrm{N} 0)$

$\mathrm{pN}(\mathrm{N} 1+\mathrm{N} 2+\mathrm{N} 3)$

14

TNM, tymor, nodes and metastasis grading system; $\mathrm{pN}$, pathological lymph-node metastasis stage.

Assay of luciferase activity. The luciferase assay was performed as previously described (10).

Cell proliferation (MTT) assay and colony formation assay. MTT and colony formation assays were performed as previously described (11)

Flow-cytometric analysis of apoptosis. An Annexin V-fluorescein isothiocyanate apoptosis detection kit (Oncogene Research Products, Boston, MA, USA) was used to detect apoptosis, as previously described (12). All samples were assayed in triplicate.

Terminal deoxynucleotidyl transferase dUTP nick end labeling (TUNEL) assay. A549 cells were transfected with either miR-338-3p mimics or miR-control. The cells were seeded on a 14-well slide 18-24 h after transfection. At 12-16 h after seeding, the cells were induced by 1 ppc $(54 \mathrm{lg} / \mathrm{ml})$ paclitaxel for 8-18 h. Cell apoptosis was detected using an in situ Cell Death Detection kit and fluorescein (Roche Applied Science, Indianapolis, IN, USA) according to the manufacturer's instructions. Subsequently, 4,6-diamidino-2-phenylindole staining was used to determine the number of nuclei and the total cell number.

Analysis of caspase-3 activity. Caspase-3 activity was determined using the colorimetric CaspACE Assay system (Promega Corporation, Madison, WI, USA) following the 


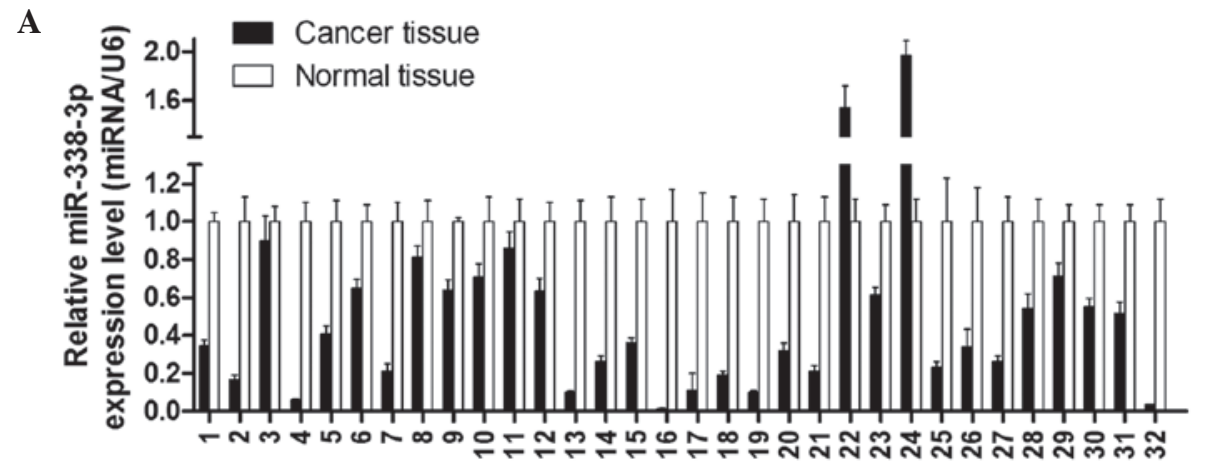

B

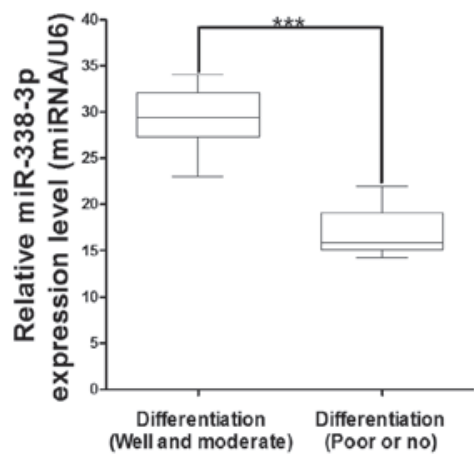

C

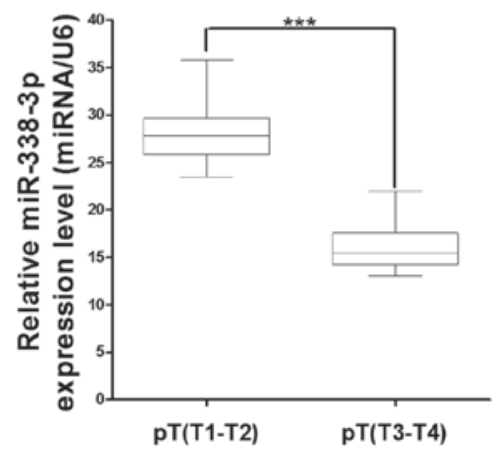

D

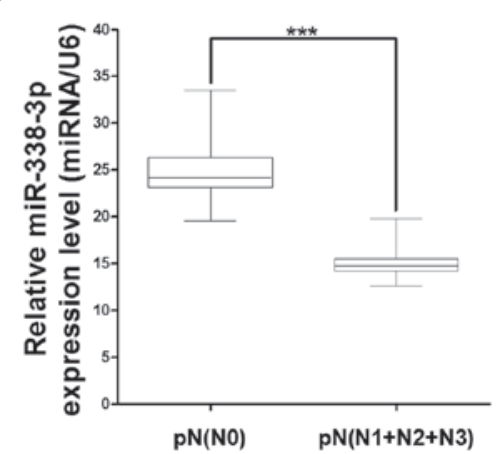

Figure 1. Determining miR-338-3p expression levels in NSCLC tissues and subsequent clinical significance. (A) Relative miR-338-3p expression levels in NSCLC tissues in comparison with those of corresponding non-tumor lung tissues. (B) miR-338-3p expression levels were significantly higher in patients with well- or moderately differentiated tumors than in those with poorly or non-differentiated tumors. (C) miR-338-3p expression levels were significantly lower in patients with tumors at a higher pathological stage than in those with tumors at a lower pathological stage. (D) miR-338-3p expression levels were significantly lower in patients with lymph-node metastasis than in those with no lymph node-metastasis. miR-338-3p expression levels were normalized to U6 expression levels.Vales are presented as mean \pm standard deviation. ${ }^{* * *} \mathrm{P}<0.001$. miR, microRNA; pT, pathological tumor stage; pN, pathological lymph-node metastasis stage; NSCLC, non-small-cel lung carcinoma.

manufacturer's instructions, as previously reported (13). Each determination was performed in triplicate.

\section{Results}

Reduced miR-338-3p expression levels in NSCLC tissues correlate with poor tumor differentiation, advanced pathological stage and lymph node metastasis. The characteristics of the 32 NSCLC patients are shown in Table I. miR-338-3p was observed to be significantly downregulated in NSCLC tissues compared with matched adjacent normal lung tissues. Among the 32 matched normal and NSCLC tumor tissues, miR-338-3p was significantly downregulated in 30 cancer tissues compared with the matched adjacent normal lung tissues (Fig. 1A). Furthermore, the correlation of miR-338-3p expression levels with clinicopathological factors was also examined (Fig. 1B, C and D). miR-338-3p downregulation was significantly correlated with poor tumor differentiation $(\mathrm{P}<0.001)$, advanced pathological stage $(\mathrm{P}<0.001)$ and lymph-node metastasis $(\mathrm{P}<0.001)$. Therefore, downregulation of miR-338-3p expression may be important in NSCLC progression and development.

miR-338-3p overexpression increases NSCLC cell in vitro proliferation. As miR-338-3p was found to be downregulated in NSCLC tissues, the expression of miR-338-3p in SPC-A1 and A549 NSCLC cells was then ectopically upregulated by miR-338-3p mimics to detect the effect of miR-338-3p overexpression. As detected by TaqMan real-time PCR assay, miR-338-3p mimics significantly upregulated the levels of miR-338-3p expression in SPC-A1 and A549 cells by 23.4and 20.2-fold, respectively ( $\mathrm{P}<0.01 ;$ Fig. $2 \mathrm{~A})$. To verify that miR-338-3p functioned as a tumor suppressor, the effect of miR-338-3p overexpression on the proliferation of NSCLC cells was determined in vitro. MTT and colony formation assays were performed to assess cell viability and proliferation. A549 and SPC-A1 cells transfected with miR-338-3p mimics were observed to grow slower than cells transfected with miR-controls (Fig. 2B and C). Fig. 2D shows that the colony numbers of A549 and SPC-A1 cells transfected with miR-338-3p mimics were significantly lower than those of cells transfected with miR-control $(\mathrm{P}<0.01)$. Thus, the results of the colony formation assay were consistent with those of the MTT assay and further indicated that ectopic miR-338-3p expression inhibited the in vitro proliferation of NSCLC cells.

Ectopic miR-338-3p expression induces apoptosis in the A549 NSCLC cell line. To determine whether the NSCLC cell growth inhibition contributed to apoptosis, flow-cytometric analysis of A549 cells was performed following transfection with miR-338-3p mimics or miR-control. In the miR-338-3p-transfected A549 cells, the rates of early (D2 quadrant) and late apoptosis/necrosis (D4 quadrant) were 8.60 and 20.7\%, respectively. By contrast, the rates of early (D2 quadrant) and late apoptosis/necrosis (D4 quadrant) in miR-control-transfected A549 cells were 1.3 and $0.6 \%$, respectively (Fig. 3A). 


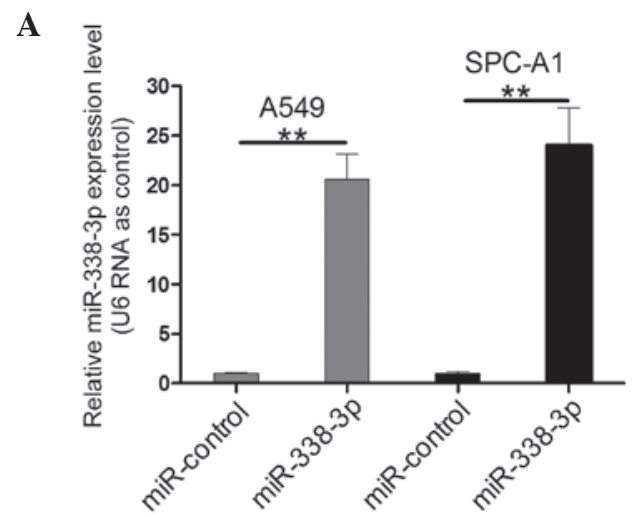

B

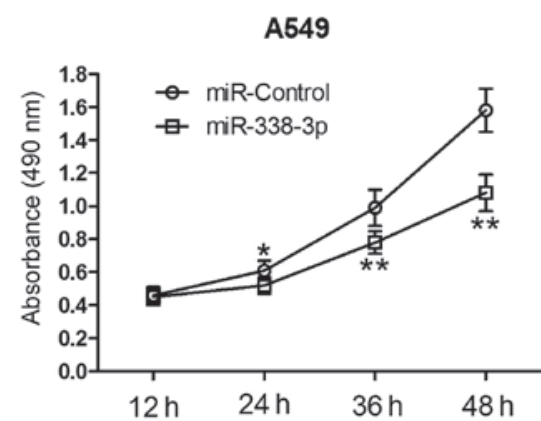

D

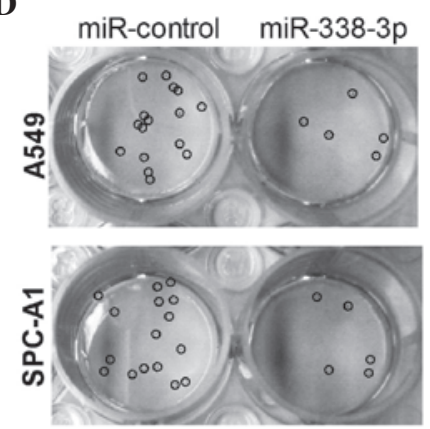

C
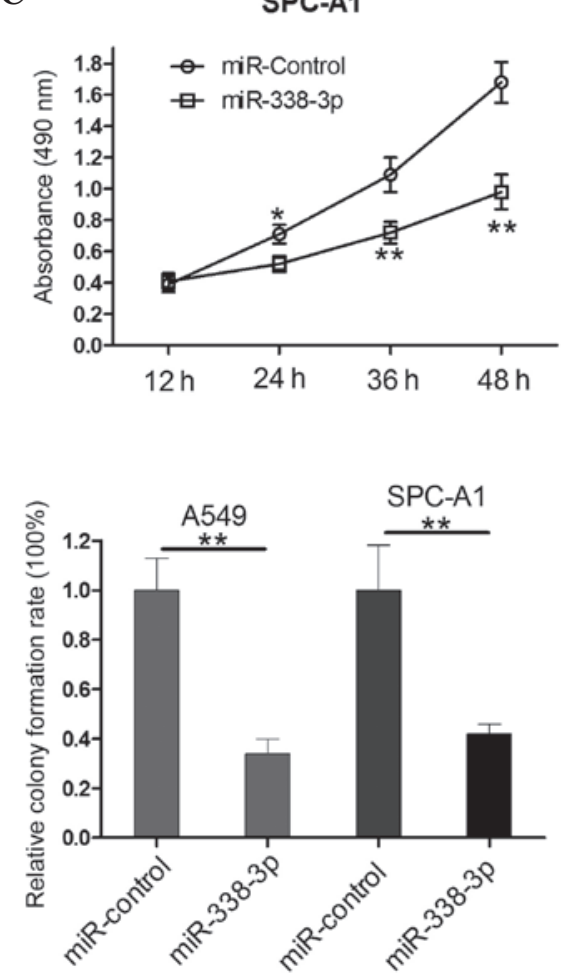

Figure 2. Overexpression of miR-338-3p inhibits in vitro growth of A549 and SPC-A1 non-small-cell carcinoma cells. The cells were transfected with miR-338-3p mimics or miR-controls, respectively. (A) Relative miR-338-3p expression levels in the two cell groups, normalized to U6 expression levels. (B and C) At $48 \mathrm{~h}$ after transfection, an MTT assay was performed to determine cell proliferation. Data are presented as the mean \pm standard deviation from three independent experiments. (D) Representative results of colony formation of A549 and SPC-A1 cells transfected with miR-338-3p mimics or miR-control. Results were determined by three independent experiments. ${ }^{*} \mathrm{P}<0.05$ and ${ }^{* *} \mathrm{P}<0.01$ as compared with the control. miR, microRNA.

Consistent with the flow cytometry results, the TUNEL assay revealed that the upregulated miR-338-3p significantly increased paclitaxel-induced apoptosis in A549 cells $(\mathrm{P}<0.01$; Fig. 3B). Furthermore, cleaved caspase-3 expression levels were detected by western blotting; caspase-3 activity was significantly higher in the cell extracts prepared from miR-338-3p mimics-transfected A549 cells than those in the miR-control-transfected cells $(\mathrm{P}<0.01 ;$ Fig. $3 \mathrm{C})$. These results suggested that the ectopic expression of miR-338-3p enhanced apoptosis in A549 cells in a caspase-3-dependent manner.

miR-338-3p directly targets the RAB14 gene by interaction with the first binding site in the 3 ' untranslated region (3'-UTR). Three miRNA target prediction algorithms (TargetScan, miRBase targets and PicTarget) were used to computationally screen for genes with miR-338-3p complementary sites in the 3'-UTR. RAB14 was found to be a putative target of miR-338-3p, with two putative target sites in the 3'UTR. To confirm this possibility, the miR-338-3p binding sequences present in the 3'-UTR of wild-type (WT) RAB14 mRNA (WT-3'-UTR), mutant site 1 (RAB14-3'-UTR-mut1) or mutant site 2 (RAB14-3'-UTR-mut2) were subcloned downstream of the luciferase reporter gene in the pGL3 vector (Fig. 4A) and then co-transfected into A549 cells. The relative luciferase activity of the reporter that contained wild-type 3'-UTR was found to be significantly reduced (by $50 \%$; $\mathrm{P}<0.05$ ) when miR-338-3p was co-transfected and was significantly increased (by $30 \%$; $\mathrm{P}<0.05$ ) when anti-miR-338-3p was co-transfected, but the luciferase activity of the RAB14-3'-UTR-mut1 reporter was unaffected by simultaneous transfection of 
A

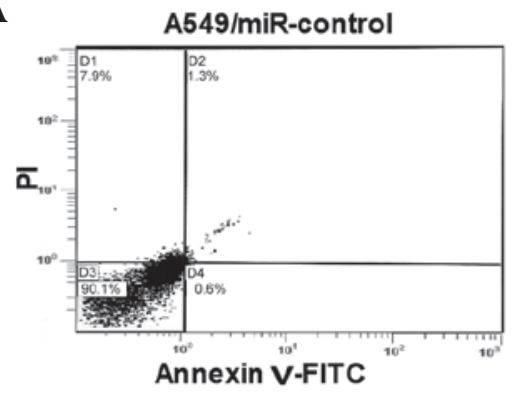

B

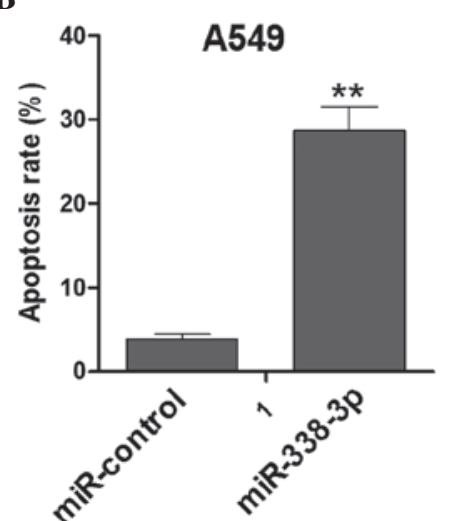

A549/miR-338-3p

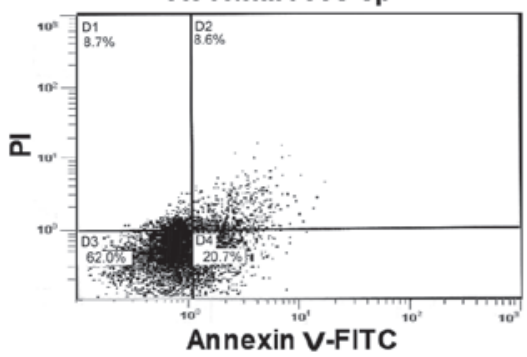

C

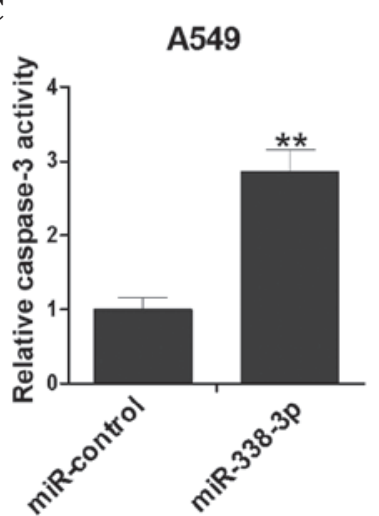

A549

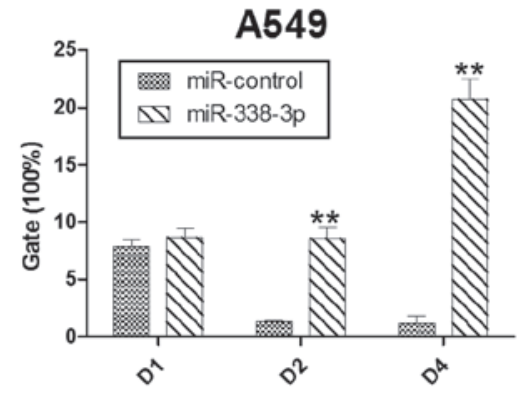

Figure 3. Ectopic miR-338-3p expression enhances apoptosis of A549 non-small-cell lung carcinoma cells. At $48 \mathrm{~h}$ after transfection with miR-338-3p or miR-control, A549 cells were collected for analysis of apoptosis. (A) Flow cytometry was used to detect the percentage of apoptotic cells in each quadrant. (B) Terminal deoxynucleotidyl transferase dUTP nick end labeling assay was used to detect the apoptotic rote of A549 cells transfected with miR-338-3p mimics or miR-control. (C) Caspase-3 activity was detected using colorimetric methods. Cleaved caspase-3 expression levels were detected by western blotting. All experiments were repeated in triplicate with similar results. ${ }^{* *} \mathrm{P}<0.01$ compared with the control group. FITC, fluorescein isothiocyanate; PI, propidium iodide; miR, microRNA; D1, necrotic cells; D2, early apoptosis quadrant; D4, late apoptosis/necrosis quadrant.

either miR-338-3p or anti-miR-338-3p. Of note, the luciferase activity of the RAB14-3'-UTR-mut2 reporter remained affected by simultaneous transfection with either miR-338-3p or anti-miR-338-3p (Fig. 4B). These results suggested that miR-338-3p may suppress RAB14 expression through the first binding site of miR-338-3p but not the second site.

PCR and western blot analyses were performed to assess whether miR-338-3p expression levels affect the expression of endogenous RAB14 at the transcriptional and translational levels. Consistent with the results of the Luciferase reporter assay, the RAB14 mRNA expression levels in the miR-338-3p mimics-transfected A549 cells were significantly reduced as compared to those in the miR-control-transfected cells, while RAB14 mRNA levels in the anti-miR-338-3p-transfected were significantly upregulated as compared with anti-miR-control-transfected cells, $(\mathrm{P}<0.05$; Fig. 4C). Furthermore, western blot analysis demonstrated that the levels of RAB14 protein expression in miR-338-3p-transfected A549 cells were significantly inhibited (by $60.8 \%$; $\mathrm{P}<0.05)$ compared with those in miR-control-transfected cells, while the levels of RAB14 protein expression in anti-miR-338-3p-transfected A549 cells were significantly upregulated (by $72 \% ; \mathrm{P}<0.05$ ) compared with those in anti-miR-control-transfected cells (Fig. 4D). These data indicated that miR-338-3p directly targeted RAB14 in NSCLC cells by interaction with the first binding site in the 3'-UTR of the RAB14 gene, which may partly indicate that miR-338-3p induces growth inhibition in NSCLC cells.

\section{Discussion}

Understanding the molecular mechanisms of cancer development is important for effective therapy. Studies have reported that deregulation of miRNA expression occurs frequently in human cancer and contributes to tumorigenesis. Therefore, investigating the function of deregulated miRNAs in cancer is crucial. Previous studies have observed miR-338-3p to be downregulated in colorectal carcinoma (14), hepatocellular carcinoma (15) and gastric cancer (16), while it was upregulated in pancreatic intraepithelial neoplasms (17). However, the expression pattern of miR-338-3p in lung cancer, particularly in NSCLC, has remained to be determined to date. miRNA arrays have previously demonstrated that miR-338-3p is downregulated in NSCLC tissues $(18,19)$; in the present study, deregulated expression of miR-338-3p was determined in NSCLC using PCR. miR-338-3p was confirmed to be significantly downregulated in NSCLC tissues (30/32 samples) compared with the corresponding matched adjacent normal tissues. The relative expression levels of miR-338-3p in the NSCLC patients were also found to be positively associated with tumor differentiation, and negatively associated with the pathological stage and the status of lymph-node metastasis. Due to the limited number of tissue samples in the present study, further investigation of a larger patient population is required to confirm the clinical significance and prognostic evaluation of miR-338-3p in NSCLC. 
A

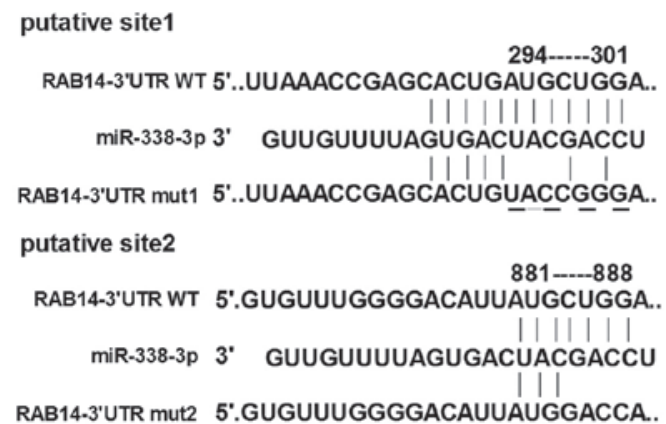

C

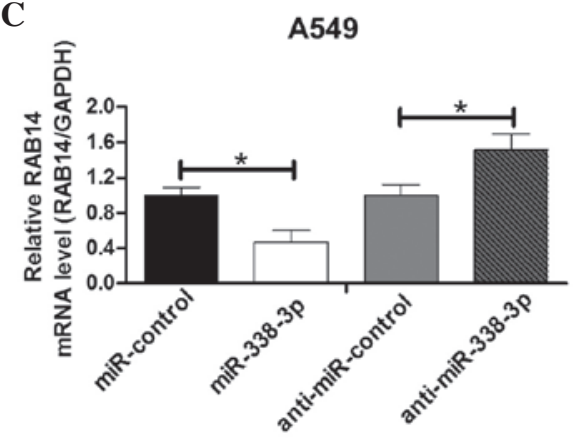

B

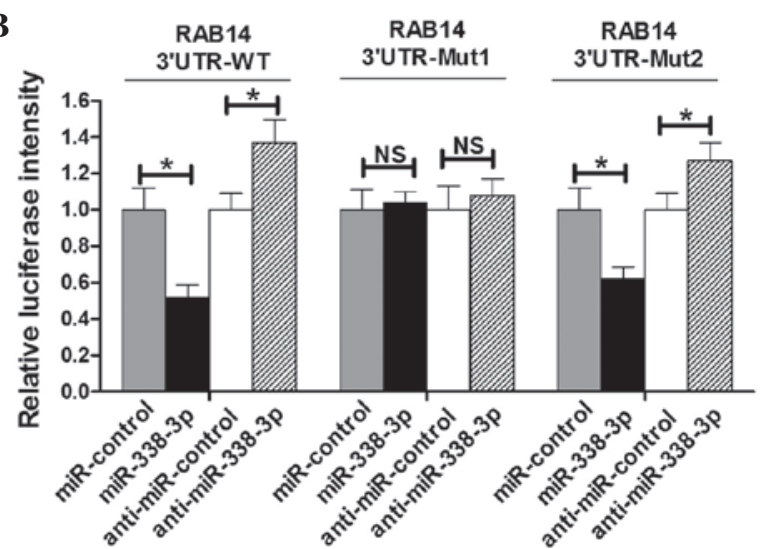

D

\section{A549}

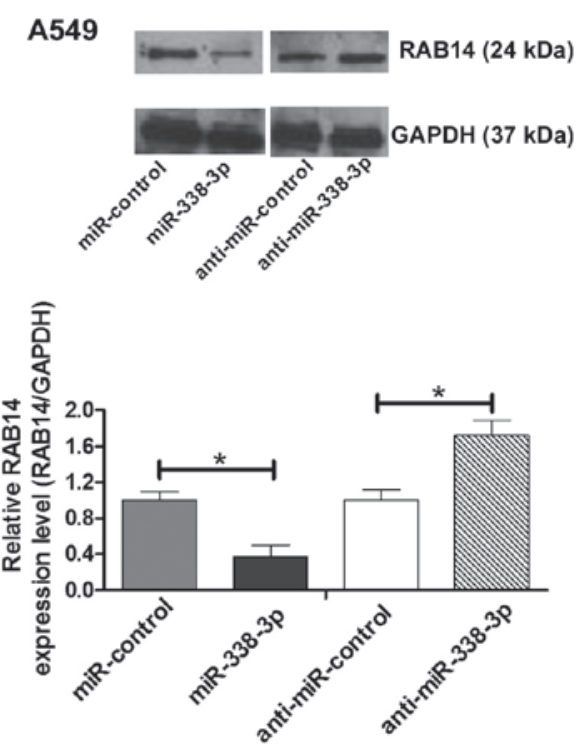

Figure 4. microRNA (miR)-338-3p directly targets RAB14 mRNA through the first binding site. (A) The two putative miR-338-3p-binding sites in the 3'-UTR of RAB14 mRNA were termed site 1 and site 2 . A mutation was generated in the RAB14 3'-UTR sequence in the complementary site for the seed region of miR-338-3p, as shown. (B) The wild-type (RAB14 3'-UTR-WT) or mutant (RAB14 3'-UTR-mut1 or mut2) reporter plasmids were co-transfected into A549 cells with miR-338-3p or miR-control. The normalized luciferase activity in the control group was set as the relative luciferase activity. (C) RAB14 mRNA expression levels were analyzed by reverse transcription polymerase chain reaction assay. GAPDH served as an internal control. (D) RAB14 protein expression levels were analyzed by western blot analysis. GAPDH served as an internal control. All experiments were repeated in triplicate with similar results. ${ }^{*}<0.05$; NS, P>0.05. miR, microRNA; 3'-UTR, 3' untranslated region; RAB14, Ras-related protein 14; WT, wild-type; mut1/2, mutant site 1/2.

A previous study has demonstrated that forced expression of miR-338-3p in colorectal cancer (CRC) cells suppressed cell proliferation and induced apoptosis, whereas inhibition of miR-338-3p in the CRC cells promoted growth (14). miR-338-3p has also been demonstrated to suppress liver cancer cell invasion (15). Therefore, miR-338-3p may also exert a tumor suppressor role in NSCLC. To analyze this in the present study, miR-338-3p expression in NSCLC cells was enhanced, and MTT, colony formation, TUNEL and flow cytometric assays were subsequently performed. Overexpression of miR-338-3p was determined to suppress NSCLC cell proliferation and induce apoptosis, which is concurrent with the role of miR-338-3p in CRC cells. However, when a Transwell invasion assay was performed, no difference between the invasive ability of overexpressed-miR-338-3p A549 cells and control A549 cells was detected (data not shown). This is not consistent with the role of miR-338-3p in liver cells, which may be explained by evidence that miRNAs function in a tissue-specific manner. For example, certain studies have indicated suppressor roles of miR-15a in various human tumor transformations, but other studies reported that miR-15a was frequently upregulated in cervical cancer (20-22). In the present study, miR-338-3p was demonstrated to suppress the proliferation of NSCLC cells and induce apoptosis in vitro, but not affect NSCLC cell invasive ability.

miRNAs may exert tumor suppressive or oncogenic roles through target oncogenes or tumor suppressor genes, thus investigating the targets of miR-338-3p in lung cancer is crucial for understanding the underlying regulatory mechanisms. In the present study, bioinformatics was used for target gene prediction. Considering the overlap of the genes identified by TargetScan, miRBase targets and PicTarget, RAB14 was selected as a potential target for further validation. RAB14 is a member of the RAS oncogene family of small GTPases, which contains $>170$ members (23). In addition to the most-widely analyzed small G protein, Ras, which is 
critical in human oncogenesis, RAB14 protein may also be involved in human cancer (24). Wang et al (11) reported that RAB14 was involved in miR-451-induced tumor suppressor effects in lung cancer. In the present study, luciferase reporter, western blotting and RT-PCR assays were performed to verify that miR-338-3p directly targets RAB14 through interaction with the first binding site in the 3'-UTR, which may explain the miR-338-3p-induced lung cancer cell growth inhibition and enhanced apoptosis.

In conclusion, the present study generated the following major findings: miR-338-3p was downregulated in NSCLC tissues, and was significantly correlated with NSCLC cancer differentiation, pathological stage and lymph-node metastasis; miR-338-3p overexpression suppressed NSCLC cell proliferation and enhanced apoptosis; and miR-338-3p directly targeted RBA14, and inhibited RBA14 expression at the mRNA and protein levels. However, further studies are required, using larger number of clinical samples for analysis of miR-338-3p expression levels in NSCLC, together with the subsequent clinical significance and prognostic evaluation. Furthermore, whether the reported RAB14 regulator miR-338-3p exerts a synergistic or antagonistic effect on the regulation of RAB14 expression requires to be elucidated.

\section{References}

1. Jemal A, Siegel R, Ward E, et al: Cancer statistics, 2008. CA Cancer J Clin 58: 71-96, 2008.

2. Verdecchia A, Francisci S, Brenner H, et al: Recent cancer survival in Europe: a 2000-02 period analysis of EUROCARE-4 data. Lancet Oncol 8: 784-796, 2007.

3. Bartel DP: MicroRNAs: genomics, biogenesis, mechanism, and function. Cell 116: 281-297, 2004.

4. Brennecke J, Hipfner DR, Stark A, Russell RB and Cohen SM: Bantam encodes a developmentally regulated microRNA that controls cell proliferation and regulates the proapoptotic gene hid in Drosophila. Cell 113: 25-36, 2003.

5. Skaftnesmo KO, Prestegarden L, Micklem DR and Lorens JB: MicroRNAs in tumorigenesis. Curr Pharm Biotechnol 8 : 320-325, 2007.

6. Ambros V: The functions of animal microRNAs. Nature 431: 350-355, 2004.

7. Shenouda SK and Alahari SK: MicroRNA function in cancer: oncogene or a tumor suppressor? Cancer Metastasis Rev 28 : 369-378, 2009.
8. Zhang B, Pan X, Cobb GP and Anderson TA: MicroRNAs as oncogenes and tumor suppressors. Dev Biol 302: 1-12, 2007.

9. Chaudhuri K and Chatterjee R: MicroRNA detection and target prediction: integration of computational and experimental approaches. DNA Cell Biol 26: 321-337, 2007.

10. Xu T, Zhu Y, Xiong Y, et al: MicroRNA-195 suppresses tumorigenicity and regulates G1/S transition of human hepatocellular carcinoma cells. Hepatology 50: 113-121, 2009.

11. Wang R, Wang ZX, Yang JS, et al: MicroRNA-451 functions as a tumor suppressor in human non-small cell lung cancer by targeting ras-related protein 14 (RAB14). Oncogene 30: 2644-2658, 2011.

12. Yang F, Sarangarajan R, Le Poole IC, Medrano EE and Boissy RE: The cytotoxicity and apoptosis induced by 4-tertiary butylphenol in human melanocytes are independent of tyrosinase activity. J Invest Dermatol 114: 157-164, 2000.

13. Yokoi S, Yasui K, Iizasa T, et al: Down-regulation of SKP2 induces apoptosis in lung-cancer cells. Cancer Sci 94: 344-349, 2003.

14. Sun K, Deng HJ, Lei ST, Dong JQ and Li GX: miRNA-338-3p suppresses cell growth of human colorectal carcinoma by targeting smoothened. World J Gastroenterol 19: 2197-2207, 2013.

15. Huang XH, Chen JS, Wang Q, et al: miR-338-3p suppresses invasion of liver cancer cell by targeting smoothened. J Pathol 225: 463-472, 2011.

16. Li P, Chen X, Su L, et al: Epigenetic silencing of miR-338-3p contributes to tumorigenicity in gastric cancer by targeting SSX2IP. PLoS One 8: e66782, 2013.

17. Yu J, Li A, Hong SM, Hruban RH and Goggins M: MicroRNA alterations of pancreatic intraepithelial neoplasias. Clin Cancer Res 18: 981-992, 2012.

18. Tan X, Qin W, Zhang L, et al: A 5-microRNA signature for lung squamous cell carcinoma diagnosis and hsa-miR-31 for prognosis. Clin Cancer Res 17: 6802-6811, 2011.

19. Vosa U, Vooder T, Kolde R, et al: Identification of miR-374a as a prognostic marker for survival in patients with early-stage nonsmall cell lung cancer. Genes Chromosomes Cancer 50: 812-822, 2011

20. Bandi N, Zbinden S, Gugger M, et al: miR-15a and miR-16 are implicated in cell cycle regulation in a Rb-dependent manner and are frequently deleted or down-regulated in non-small cell lung cancer. Cancer Res 69: 5553-5559, 2009.

21. Bonci D, Coppola V, Musumeci M, et al: The miR-15a-miR-16-1 cluster controls prostate cancer by targeting multiple oncogenic activities. Nat Med 14: 1271-1277, 2008.

22. Wang X, Tang S, Le SY, et al: Aberrant expression of oncogenic and tumor-suppressive microRNAs in cervical cancer is required for cancer cell growth. PLoS One 3: e2557, 2008.

23. Takai Y, Sasaki T and Matozaki T: Small GTP-binding proteins. Physiol Rev 81: 153-208, 2001.

24. Agarwal R, Jurisica I, Mills GB and Cheng KW: The emerging role of the RAB25 small GTPase in cancer. Traffic 10: 1561-1568, 2009. 